\title{
Polymeric Electron-Selective Contact for Crystalline Silicon Solar Cells with an Efficiency Exceeding 19\%
}

Wenbo Jil ${ }^{1,2,3}$, Thomas Allen ${ }^{4}$, Xinbo Yang ${ }^{4}$, Guosong Zeng ${ }^{5}$, Stefaan De Wolf ${ }^{4}$, Ali Javey $^{1,2, *}$

${ }^{1}$ Electrical Engineering and Computer Sciences, University of California, Berkeley, California 94720, USA

${ }^{2}$ Materials Sciences Division, Lawrence Berkeley National Laboratory, Berkeley, California 94720, USA

${ }^{3}$ Materials Science and Engineering, University of California, Berkeley, California 94720, USA

${ }^{4}$ KAUST Solar Center (KSC), King Abdullah University of Science and Technology (KAUST), Thuwal 23955-6900, Saudi Arabia

${ }^{5}$ Chemical Sciences Division, Lawrence Berkeley National Laboratory, Berkeley, California 94720, USA

\section{Corresponding Author}

ajavey@berkeley.edu 


\section{ABSTRACT}

Carrier-selective contacts have become a prominent path forward towards efficient crystalline silicon (c-Si) photovoltaics. Among the proposed contacting materials, organic materials may offer simplified and low-cost processing compared with typical vacuum deposition techniques. Here, branched polyethyleneimine (b-PEI) is presented as an electron-transport layer (ETL) for c-Si solar cells. The incorporation of b-PEI interlayer between $\mathrm{c}-\mathrm{Si}(n)$ and $\mathrm{Al}$ leads to a low contact resistivity of $24 \mathrm{~m} \Omega \mathrm{cm}^{2}$. Silicon heterojunction solar cell integrated with b-PEI is demonstrated achieving a power conversion efficiency of $19.4 \%$, which improves the benchmark efficiency of a c-Si solar cell with an organic ETL. This electron-selectivity of b-PEI is attributed to its Lewis basicity, i.e., electron donating ability, promoting favorable band bending at the c-Si surface for electron transport. Moreover, several other Lewis base polymers perform as efficient ETLs in organic/c-Si hybrid devices, indicating Lewis basicity could be a guideline for future organic ETLs design.

\section{TOC GRAPHICS}

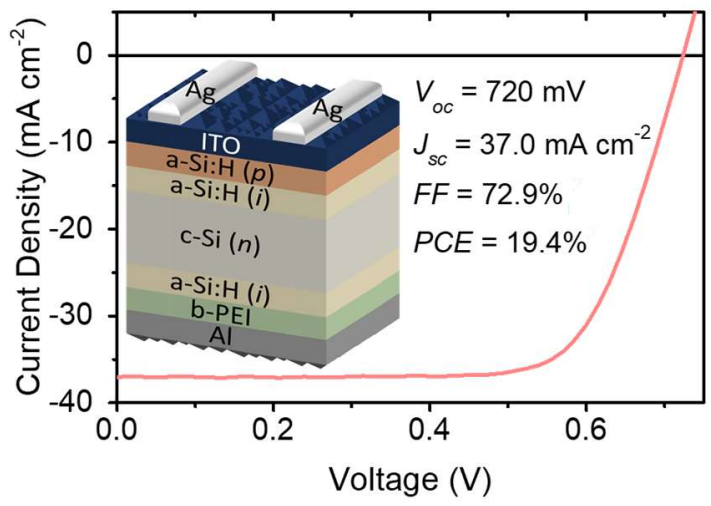


Research on carrier-selective contacts for crystalline silicon (c-Si) photovoltaics (PV) has achieved great progress in recent years, with an expanded library of available electron and hole transport layers (ETLs and HTLs), offering opportunities for improved understanding of device physics as well as enabling efficient solar cells with low-cost, scalable materials and processes. ${ }^{1}$ Different classes of materials, such as oxides, halides, nitrides and organic molecules, have been demonstrated as ETLs and HTLs on pristine and/or passivated c-Si surfaces. ${ }^{2-9}$ Compared with conventional PV technologies, the implementation of full-area carrier-selective contacts eliminates the high temperature diffusion and contact patterning steps, resulting in a simplified fabrication processes. In addition, many carrier-selective contact materials have the potential to mitigate or eliminate the fundamental physical loss mechanisms that constrain the power conversion efficiency (PCE) of c-Si solar cells fabricated with conventional contacting schemes (e.g. parasitic free carrier absorption, Auger recombination and band narrowing). ${ }^{10-12}$ Organic molecules offer a wide variety of candidate materials for selective contacts thanks to their adjustable functional group modification, and offer great promise for low-cost fabrication. ${ }^{13}$ Among the organic materials, poly(3,4-ethylene dioxythiophene):poly(styrenesulfonate) (PEDOT:PSS) has received the increasing interests in hybrid organic/c-Si solar cells due to its relatively high work function (5.0-5.1 eV), passivation effect on c-Si surfaces and transparent conductive properties. ${ }^{8,14-16}$ By using PEDOT:PSS as front and rear side HTL, c-Si solar cells have been reported with the promising PCEs of above $16 \%$ and $20 \%$ respectively. ${ }^{8,17}$

As for the electron-selective contact, a simple, directly metallized c-Si( $n) / \mathrm{Al}$ contact is well known to exhibit a large energy barrier for electrons as well as severe carrier recombination because of the high concentration of surface defects, including metal induced gap states. ${ }^{18-}$ 
${ }^{20}$ Therefore, an efficient electron-selective contact is also required in the design of hybrid organic/c-Si solar cells. Several types of organic molecules have been demonstrated as ETLs for c-Si cells, such as PEO (poly(ethylene oxide)), Liq (8-hydroxyquinolinolato lithium), CPTA ( $\mathrm{C}_{60}$ pyrrolidine tris-acid) and amino acids, among which L-histidine, an amino acid, is used as an electron-selective contact of c-Si $(n)$ cells with a PCE of $17.9 \%$. ${ }^{21-25}$ Common to all of these devices is a relatively low open-circuit voltage $\left(V_{o c}\right)$, due to poor surface passivation, which hinders the PCE of hybrid organic/c-Si solar cells with organic ETLs. The search for an organic ETL that is compatible with a well-passivated c$\mathrm{Si}$ architecture (e.g., $\mathrm{SiO}_{2} / \mathrm{c}-\mathrm{Si}$, a-Si:H/c-Si) has been elusive to date, motivating the present work.

Polyethyleneimine (PEI) is a polymer containing a high concentration of amine groups. Lone pairs of electrons from nitrogen atoms in PEI contribute to its relatively strong Lewis basicity, i.e., its electron donating capability. Previously PEI was reported as an effective ETL for organic and perovskite solar cells, organic light-emitting diodes as well as an $n$ type dopant in electronic devices. ${ }^{26-31}$ Despite this wide range of applications, the study of PEI on c-Si solar cells as electron contact has not been reported yet. Herein, we demonstrate that branched PEI (b-PEI) leads to a low contact resistivity in c-Si(n)/b-PEI/Al electronselective contact stacks. By implementation of b-PEI as ETL in silicon heterojunction solar cells, a champion PCE of $19.4 \%$ is achieved. This PCE is first to be reported for a hybrid organic/c-Si device exceeding 19\% using an organic ETL. Our further study of several other Lewis base polymers indicates a relationship between Lewis base strengths and ETL characteristics, which could be a guideline for the future contact design of hybrid organic/cSi solar cells. 
The b-PEI ethanol solution was spin-coated on a polished $1 \Omega \mathrm{cm} \mathrm{c}-\operatorname{Si}(n)$ wafer, yielding b-PEI layers with thickness of 2-10 $\mathrm{nm}$. Spin-coating was performed under identical conditions for all samples, where the film thickness was controlled by using different solution concentrations ranging from 0.01 to $0.10 \mathrm{wt} . \%$ (details in experimental section). The morphology of b-PEI was characterized by atomic force microscopy (AFM), shown in Figure $\mathrm{S} 1$. We found that the thin layer is continuous, featuring a surface roughness $\mathrm{R}_{\mathrm{a}}$ of $0.19 \mathrm{~nm}$, which is much smaller than its thickness $(>2 \mathrm{~nm})$. Aluminum (Al) was thermally evaporated in the subsequent step as the electrode for $I-V$ measurements. The $I$ $V$ characteristics versus b-PEI thickness was studied, featuring a contact structure in Figure 1a. Compared with the $\mathrm{c}-\mathrm{Si}(n) / \mathrm{Al}$ contact, resistance significantly decreases after incorporating a $2 \mathrm{~nm}$-thick b-PEI interlayer between c-Si $(n)$ and $\mathrm{Al}$. The contact becomes resistive when the b-PEI thickness is $\sim 6$ and $10 \mathrm{~nm}$, indicating an electron-tunneling mechanism through b-PEI. The contact resistivity $\rho_{c}$, determined by the transfer length method (TLM), is extracted as $24 \mathrm{~m} \Omega \mathrm{cm}^{2}$ from the $2 \mathrm{~nm}$ b-PEI/Al stack in Figure $1 \mathrm{~b}$. With the addition of a PECVD deposited intrinsic hydrogenated amorphous silicon a-Si:H(i) passivating interlayer, the $\mathrm{c}-\mathrm{Si}(n) / \mathrm{a}-\mathrm{Si}: \mathrm{H}(i) / \mathrm{b}-\mathrm{PEI} / \mathrm{Al}$ heterocontact is found to remain Ohmic, with $\rho_{\mathrm{c}}=156 \mathrm{~m} \Omega \mathrm{cm}^{2}$ (see Figure S2). Such a-Si:H(i) passivating interlayer is typically employed in silicon heterojunction solar cells, inserted between wafer and ETL or HTL, resulting in very high operating voltages for such devices. ${ }^{32}$ The a-Si:H(i) films we used here were optimized for such purposes. We investigated the effect of the b-PEI layer on the a-Si:H(i) passivation quality by effective carrier lifetime measurement, which is shown in Figure 1c. The red circles and black squares represent the injection-leveldependent effective carrier lifetime of $3 \Omega \mathrm{cm}$ c-Si $(n)$ wafer with double-side a-Si:H $(i)$ 
passivation before and after $3 \mathrm{~nm}$-thick b-PEI capping, respectively. The results show the effective carrier lifetime (at the injection level of $10^{15} \mathrm{~cm}^{-3}$, corresponding to typical solar cell operating conditions under 1 sun illumination) keeps constant before and after b-PEI coating, featuring a high value of 2.4 milliseconds and an implied $V_{o c}$ of $725 \mathrm{mV}$.

a

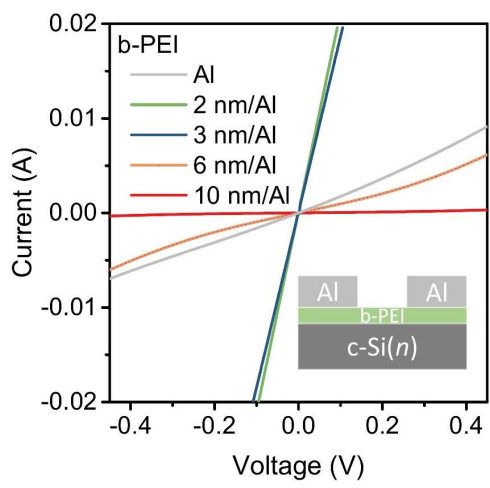

b

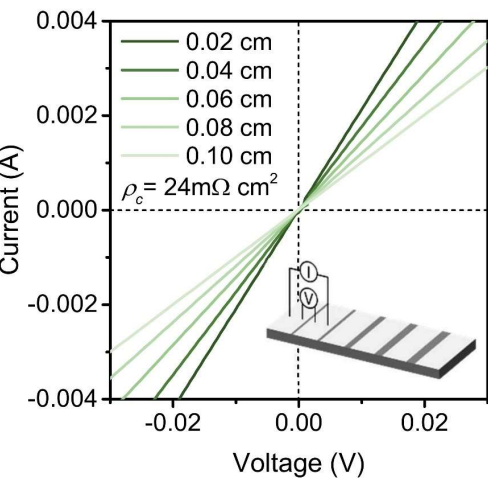

$\mathrm{C}$

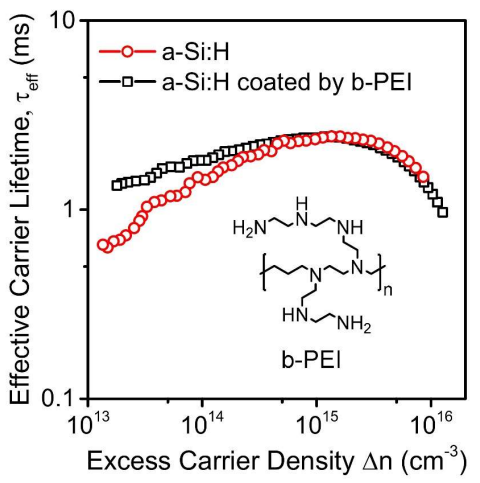

Figure 1. a) $I-V$ characteristic of the b-PEI/Al stack on $1 \Omega \mathrm{cm}$ c-Si $(n)$. The thickness of b-PEI layer ranges from $0-10 \mathrm{~nm}$ and inset is the cross-section of structure for measurement. b) Extracted contact resistivity $\left(\rho_{c}\right)$ of $2 \mathrm{~nm} \mathrm{~b}$-PEI/Al heterocontact on $n$ type $1 \Omega \mathrm{cm}$ silicon wafers. The inset shows the TLM structure for $\rho_{c}$ extraction. c) Effective carrier lifetime $\tau_{\text {eff }} v s$. excess carrier density of a-Si:H $(i)$ passivated c-Si $(n)$ before and after b-PEI coating. The molecular structure of b-PEI is shown.

Materials-based characterizations of b-PEI was then performed. An $80 \mathrm{~nm}$-thick b-PEI layer was spin-coated on fused $\mathrm{SiO}_{2}$ substrate for optical transmittance and absorption measurements. Its Tauc plot is provided in Figure 2a with the extracted bandgap of 6.15 $\mathrm{eV}$; its large value is in line with the result of resistance $v s$. thickness in Figure 1a. The inset of Figure 2a plots the transmittance of b-PEI from 180 to $1400 \mathrm{~nm}$, which includes the absorption spectra of c-Si solar cells. Its transmittance is close to $100 \%$ in most of the 
measured range and starts to decrease when the wavelength is shorter than $300 \mathrm{~nm}$, showing that b-PEI possesses higher transparency than conventional a-Si:H( $n)$ contact. The high-resolution X-ray photoelectron spectroscopy (XPS) spectra of N 1s from different thickness b-PEI on c-Si(n) substrate is collected (Figure S3). The predominant peak of $399.1 \mathrm{eV}$ is from the amine groups of b-PEI when the film thickness is $\sim 80 \mathrm{~nm} \cdot{ }^{33}$ A peak of $400.3 \mathrm{eV}$ appears when we collected signal from 3 and $12 \mathrm{~nm}$-thick b-PEI layers, assigned to positively charged $\mathrm{N}$ atoms in b-PEI. ${ }^{34}$ This peak can be mainly interpreted as the charge transfer at the b-PEI/c-Si $(n)$ interface which leads to a positively charged b-PEI layer. The work function of b-PEI/Al contact was obtained from the secondary electron cutoff. Figure $2 \mathrm{~b}$ shows this contact has a low work function of $2.93 \mathrm{eV}$, which is the reason for Ohmic contact and low resistivity on pristine and a-Si:H(i) passivated c-Si $(n)$, as demonstrated for other inorganic low work function ETLs. ${ }^{5,35}$ The energy band diagram is schemed as Figure 2c. As a Lewis base, b-PEI has strong tendency to donate electrons, resulting in a positively charged layer, corresponded to the measured low work function. We estimate the upper limit of positive charge density in b-PEI interlayer equal to $5 \times 10^{12}$ $\mathrm{cm}^{-2}$ as a simple guideline by utilizing assumptions discussed in previous research. ${ }^{36}$ Due to the charge neutrality principle, electrons in c-Si accumulate at the interface. The energy band of c-Si bends downward at this interface and electrons can be transported from c-Si to $\mathrm{Al}$ with a low barrier height. 

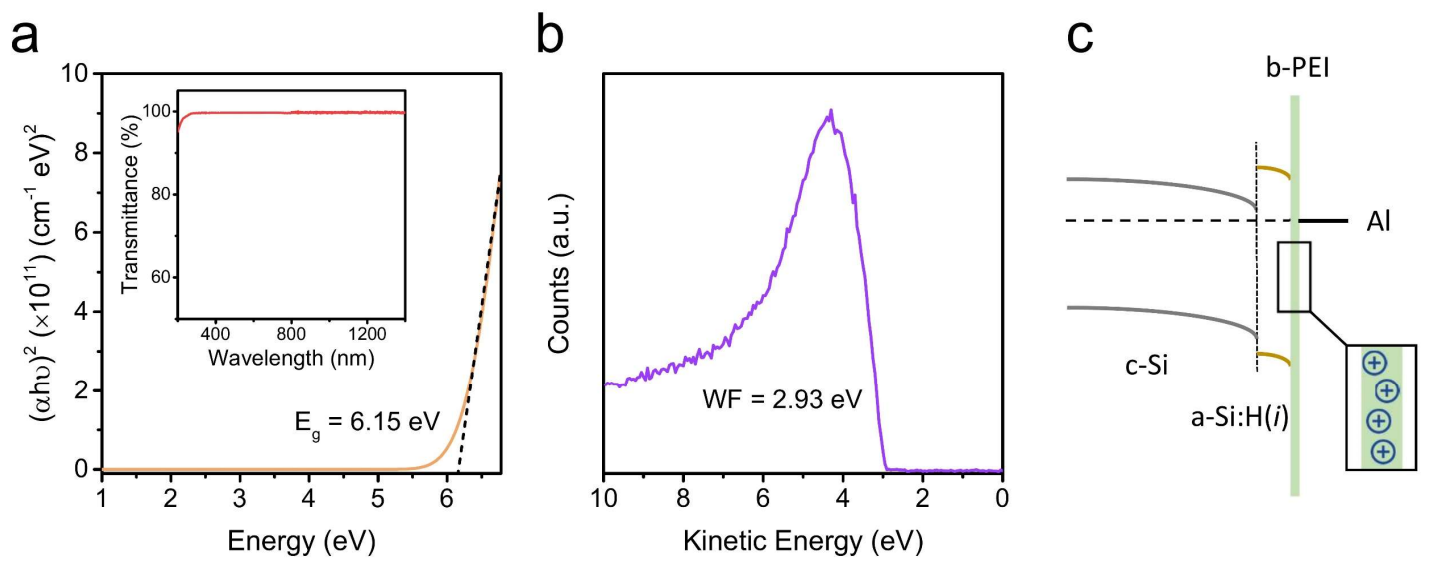

Figure 2. a) Tauc plot for b-PEI layer showing an optical bandgap ( $\left.E_{g}\right)$ of $\sim 6.15 \mathrm{eV}$. The inset shows the transmittance measurement ranging from 180 to $1400 \mathrm{~nm}$. b) Low work function (WF) of b-PEI/Al stack yielded by the secondary electron cutoff. c) Schematic of energy band diagram for b-PEI/Al as heterocontact implementing a-Si:H $(i)$ as interlayer, the right panel showing a zoom-in of positive charges in b-PEI layer.

The b-PEI/Al contact is finally integrated into a $2 \times 2 \mathrm{~cm}^{2}$ silicon heterojunction cell, the architecture of which is sketched in Figure 3a. This device features a random pyramid textured front surface, capped with a stack of $i / p$ a-Si:H as passivating hole-selective contact at the front side. Onto this HTL, an indium tin oxide (ITO) layer was sputtered and grid metallization was applied. The b-PEI layer was then spin-coated on the textured and a-Si:H(i)-passivated rear side with different thickness, followed by thermal evaporation of $\mathrm{Al}$ as full-area rear electrode. The $J-V$ curves measured under 1-sun illumination are shown in Figure 3b. The champion PCE reaches $19.4 \%$ in the optimal $3 \mathrm{~nm}$ b-PEI cell, which is the new benchmark for hybrid organic/c-Si solar cells using an organic ETL. This device is characterized by $V_{o c}, J_{s c}$ and fill factor $(F F)$ values of $720 \mathrm{mV}, 37.0 \mathrm{~mA} \mathrm{~cm}^{-2}$ and $72.9 \%$ respectively. The series resistances of cells with 2 and $3 \mathrm{~nm}$-thick b-PEI are extracted as 2 
and $2.9 \Omega \mathrm{cm}^{2}$ respectively, which could be further decreased by modifying b-PEI with functional groups to increase its electron conductivity. The $J-V$ curves of the fabricated cells exhibit an 'S-shape' when the coated b-PEI layer is thicker than $3 \mathrm{~nm}$, consistent with the increasing contact resistance of b-PEI/Al stack in Figure 1a. As b-PEI layer becomes thicker, this parasitic resistance from the contact also undermines the $V_{o c}$ of cells. Figure $3 c$ shows the measured external quantum efficiency (EQE), reflection, and internal quantum efficiency (IQE) of the champion device. All these measurements were taken inbetween the Ag metal grid fingers. Inset is photograph of the fabricated cell with a contact fraction of $\sim 5 \%$. The integration of the EQE weight against the AM 1.5 solar spectrum product gives a $J_{s c}=38.8 \mathrm{~mA} \mathrm{~cm}{ }^{-2}$ without contact fraction correction and $36.9 \mathrm{~mA} \mathrm{~cm}{ }^{-2}$ after correction, which is in line with the $J_{s c}$ derived from $J-V$ measurements. The relatively low EQE in the short wavelength region is attributed to the relatively strong parasitic absorption of the $i / p$ a-Si:H stack. ${ }^{10}$ This implies that a higher $J_{s c}$ and PCE can be achieved by utilizing hole-selective contacts with a better optical transparency.

a

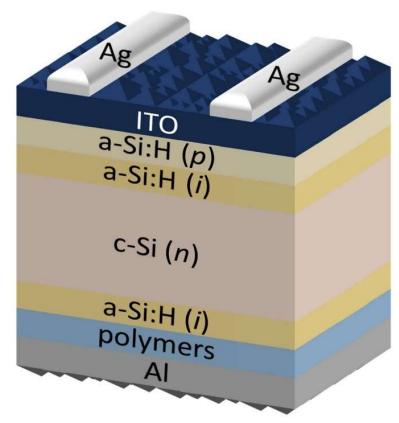

b

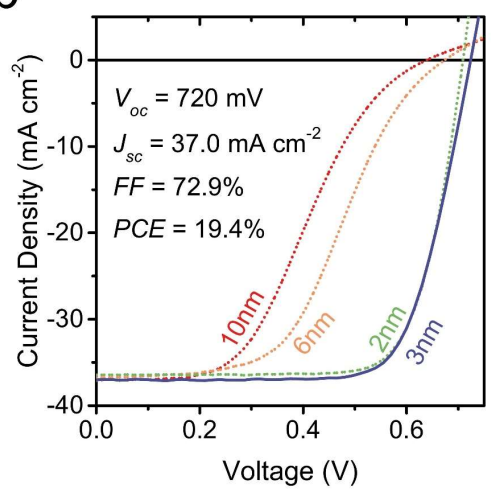

C

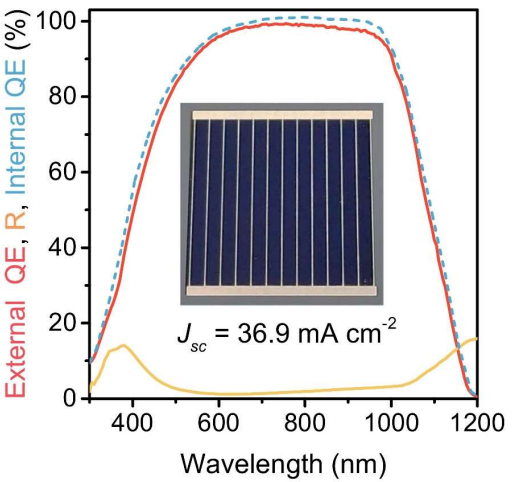

Figure 3. a) Architecture of an $n$-type silicon heterojunction cell integrating with b-PEI layer. b) $J-V$ characteristics of $n$-type heterojunction cell with different thickness of b-PEI layers collected under standard 1-sun conditions. The labelled film thickness refers to the 
previous thickness on planar wafer due to the difficulty of measuring polymer thickness on a textured surface. c) Wavelength-dependent external QE, reflection and internal QE for the champion device. The integrated $J_{s c}$ (after contact fraction correction) is calculated and the photograph of the cell front design is shown in inset.

The effect of Lewis basicity on c-Si solar cell performance was also further studied. Several commercial Lewis base polymers were tested on silicon heterojunction cells, molecular structures of which are shown in Figure 4a. The Lewis basicity of linear PEI (1-PEI), polyethyleneimine ethoxylated (PEIE) and poly(4-vinylpyridine) (P4VP) mainly originate from the paired electrons of nitrogen in amine groups and pyridine N. Polyethylene glycol (PEG) and poly(vinyl alcohol) (PVA) show basicity due to the paired electrons of oxygen in ether and hydroxyl group. All of these polymers were dissolved in ethanol with a concentration of $0.02 \mathrm{wt} . \%$ and spin-coated via the same recipe used to fabricate the cells with b-PEI layer. The illuminated $J-V$ curves are plotted in Figure $4 \mathrm{~b}$. The cells implemented with 1-PEI, PEIE and P4VP layers achieve PCEs ranging from 17.9\% to $19.4 \%$, the $V_{o c}$ of which are all $>690 \mathrm{mV}$, demonstrating effective electron collection by these Lewis base polymers. The cells coated by PVA and PEG are less effective and exhibit lower PCEs of approximate $15 \%$ with a $V_{o c}$ of $\sim 590 \mathrm{mV}$. The $J_{s c}$ difference results from the different optical properties and thickness of the polymers. For comparison, the light grey curve refers to thermally evaporated Al electrode without a polymer interlayer, featuring a very low $V_{o c}(<300 \mathrm{mV})$ due to metal-induced recombination as well as a poor electron-selectivity. Noticeably, it is well-known that amine and pyridine are stronger than ether and hydroxyl in terms of basicity. ${ }^{37}$ PEG and PVA as weaker Lewis bases will not induce enough downward band bending of c-Si for efficient electron collection. Therefore, 
devices with PEG and PVA show lower $V_{o c}$. These results indicate that the Lewis base strengths of organic ETLs can be an important factor related to the performance of electronselective contacts in c-Si solar cells.

a

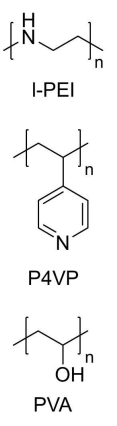

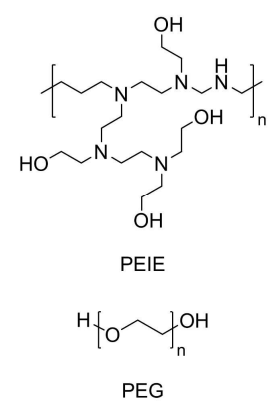

b

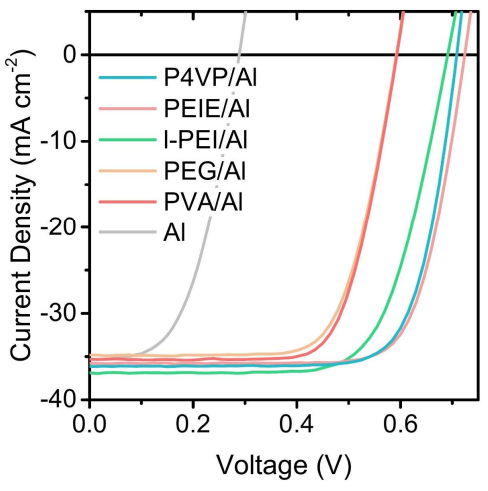

Figure 4. a) Molecular structures of tested commercial Lewis base polymers. b) $\mathrm{J}-\mathrm{V}$ characteristics of $n$-type heterojunction cell with different Lewis base polymers as organic ETLs. Al (grey curve) is fabricated without any polymer interlayer as comparison.

In this study, we have demonstrated b-PEI as an effective organic ETL that can be integrated into an a-Si:H(i) passivating electron-selective contact for c-Si solar cells. The highly transparent spin-coated b-PEI enables low contact resistivity with $\mathrm{Al}$ on c-Si $(n)$. The device with an electron-selective contact comprised of a-Si:H(i)/b-PEI/Al show an PCE up to $19.4 \%$, which improves the benchmark PCE demonstrated in a hybrid organic/c-Si solar cell with an organic ETL. The $V_{o c}$ is $>700 \mathrm{mV}$, indicating a low surface recombination velocity and efficient electron collection. The electron-selectivity of the b-PEI contact is attributed to the relatively strong Lewis basicity of b-PEI, resulting in a positively charged b-PEI layer and favorable downward band bending of $\mathrm{c}-\mathrm{Si}$, in line with a measured low work function of $2.93 \mathrm{eV}$. Importantly, several other polymers with different Lewis basicity 
also show a similar performance on c-Si solar cells, indicating a generality of this concept. Future study on associating the Lewis base strengths $v s$. device performance may yield a universal guideline for organic electron contact design in c-Si solar cells, and may even be expanded to inorganic contacts.

\section{Methods}

Chemicals and Solution Preparation: Branched polyethyleneimine solution (b-PEI) (50 wt. $\%$ in $\mathrm{H}_{2} \mathrm{O}$, average $M_{\mathrm{w}} \sim 750,000$ by LS), linear polyethyleneimine (1-PEI) (average $M_{\mathrm{n}}$ 5,000), polyethyleneimine $80 \%$ ethoxylated solution (PEIE) (37 wt.\% in $\mathrm{H}_{2} \mathrm{O}$ ), poly(4vinylpyridine) (P4VP) (average $M_{\mathrm{w}} \sim 60,000$ ), poly(ethylene glycol) (PEG) (average $M_{\mathrm{n}}$ $20,000)$ and poly(vinyl alcohol) (PVA) $\left(M_{\mathrm{w}} 13,000-23,000,87-89 \%\right.$ hydrolyzed) were purchased from Sigma-Aldrich. Branched polyethyleneimine was diluted to 0.01-0.10 wt.\% solutions by ethanol. Other polymers were dissolved/diluted to $0.02 \mathrm{wt} \%$ solutions by ethanol.

Contact and Materials Characterization: Contact resistivity samples were fabricated on the $1 \Omega \mathrm{cm} n$-type (100) c-Si wafer. After a diluted hydrofluoric acid (HF) dip for native oxide removal, the wafer was spin-coated by b-PEI with the recipe of $4000 \mathrm{rpm}$ for $60 \mathrm{~s}$. The thickness of b-PEI layer was controlled by using different concentration of solutions, measured by ellipsometer (J. A. Woollam M-2000, analyzed by Cauchy model). The solution concentration of $0.01,0.02,0.05$ and $0.10 \mathrm{wt} . \%$ corresponds to the b-PEI layer thickness of $\sim 2,3,6$ and $10 \mathrm{~nm}$ respectively. All the above steps were processed under ambient condition. Subsequently, Al $(\sim 150 \mathrm{~nm})$ was deposited by thermal evaporation 
through a shadow mask in order to obtain a transfer-length-method (TLM) pattern. I- $V$ characteristics with different pad spacing were done by Keithley 2400 SourceMeter and Agilent $4155 \mathrm{C}$. Contact resistivity on passivated $1 \Omega \mathrm{cm} \mathrm{c}-\mathrm{Si}(n)$ was extracted by using the same method. The parallel resistance through the b-PEI layer was assumed to be negligible since it was thin and insulating. Lifetime samples were prepared on $3 \Omega \mathrm{cm} n$-type c-Si wafers with random-pyramid textured. After standard Radio Corporation of America (RCA) cleaning and dipping in a dilute $\mathrm{HF}$ solution, a-Si:H(i) layers were deposited in an INDEOtec Octopus II PECVD cluster tool at $200{ }^{\circ} \mathrm{C}$. The b-PEI layer was spin-coated on this passivated wafer by using $0.02 \mathrm{wt} . \%$ solution and the same recipe as before. Effective carrier lifetime was measured by a Sinton WCT 120 photoconductance tester in transient mode. Optical measurements were made by Agilent Cary 5000 UV-Vis-NIR spectrometer. AFM for the surface morphology of b-PEI layer was conducted by a Dimension ICON AFM (Bruker, Germany) under ambient conditions. The XPS results were collected by a Kratos AXIS Ultra DLD system with a monochromatic Al Ka X-ray source and a hemispherical analyzer. Thin films of b-PEI were spin-coated on polished c-Si $(n)$ wafer for XPS characterization. The XPS data was plotted with the C 1s correction. Work function was extracted from the secondary electron cutoff by UPS mode and an added bias in this XPS system. Sample for work function was prepared as b-PEI/Al stack on c-Si(n) wafer, within which $\mathrm{Al}$ was patterned by shadow mask. The measurement was conducted at the edge of $\mathrm{Al}$ pad to observe the work function of the stack.

Cell Fabrication and Characterization: The $2 \times 2 \mathrm{~cm}^{2} n$-type cells were fabricated on high quality float-zone c-Si $(n)$ substrates $(1 \Omega \mathrm{cm}, \sim 250 \mu \mathrm{m})$. After random pyramid texturing in an alkaline solution and a standard RCA cleaning, a stack of intrinsic and boron-doped 
a-Si:H $(i / p$ a-Si:H) was deposited on the front side of the wafer utilizing hydrogen, silane and trimethyl boron as the input gases. An a-Si:H(i) passivation layer was deposited on the rear side after flipping. All the a-Si:H layers were deposited in an INDEOtec Octopus II PECVD cluster tool at $200{ }^{\circ} \mathrm{C}$. Then front transparent conductive window layer, ITO (75 $\mathrm{nm}$ ), was capped by sputtering through a shadow mask, defining the active cell area. The front metallization was prepared by screen-printing using a low-temperature Ag paste and curing at $200{ }^{\circ} \mathrm{C}$ for 15 mins in air. The rear side was treated by diluted HF and then was capped by b-PEI via spin-coating at $4000 \mathrm{rpm}$ for $60 \mathrm{~s}$. Then $\mathrm{Al}(\sim 150 \mathrm{~nm})$ was thermally evaporated on the top of b-PEI layer. The cells integrated with 1-PEI, PEIE, P4VP, PEG and PVA were fabricated by same spin-coating recipe and processing steps. The 1-sun $J$ $V$ measurement was performed by a solar simulator under standard conditions $(100 \mathrm{~mW}$

$\mathrm{cm}^{-2}, 25^{\circ} \mathrm{C}, \mathrm{AM} 1.5 \mathrm{G}$ spectrum) and an aperture mask to avoid peripheral absorption. The quantum efficiency analysis was made by an Enlitech QE-R solar cell quantum efficiency measurement system. The external quantum efficiency (EQE), reflection and internal quantum efficiency (IQE) were measured in-between Ag grid fingers.

\section{Supporting Information}

The Supporting Information is available free of charge on the ACS Publications website at DOI: XXXX.

AFM morphology characterization of b-PEI layer, extracted contact resistivity $\left(\rho_{c}\right)$ of b$\mathrm{PEI} / \mathrm{Al}$ heterocontact on a-Si:H(i)/c-Si( $n)$, high-resolution XPS spectra of $\mathrm{N} 1 \mathrm{~s}$ from b-PEI layers. 


\section{Author Information}

Corresponding Author

*E-mail: ajavey@,berkeley.edu.

\section{ORCID}

Wenbo Ji: 0000-0002-7913-361X

Xinbo Yang: 0000-0002-0622-5417

Stefaan De Wolf: 0000-0003-1619-9061

Ali Javey: 0000-0001-7214-7931

Notes

The authors declare no competing financial interest

\section{Acknowledgements}

The authors would like to thank Zhen Yuan for the help of electrical measurements. Materials characterization was supported by the Electronic Materials Programs, funded by the Director, Office of Science, Office of Basic Energy Sciences, Material Sciences and Engineering Division of the US Department of Energy under Contract No. DE-AC0205CH11231. XPS characterization was performed at the Joint Center for Artificial Photosynthesis, supported through the Office of Science of the US Department of Energy under Award Number DE-SC0004993. Work at the Molecular Foundry was supported by 
the Office of Science, Office of Basic Energy Sciences, of the US Department of Energy under the Contract No. DE-AC02-05CH11231. Device fabrication was funded by King Abdullah University of Science \& Technology under the Contract No. OSR-2017-GRGG3383.01 .

\section{References}

[1] Allen, T. G.; Bullock, J.; Yang, X.; Javey, A.; De Wolf, S. Passivating Contacts for Crystalline Silicon Solar Cells. Nat. Energy 2019, 4, 914-928.

[2] Battaglia, C.; De Nicolas, S. M.; De Wolf, S.; Yin, X.; Zheng, M.; Ballif, C.; Javey, A.

Silicon Heterojunction Solar Cell with Passivated Hole Selective $\mathrm{MoO}_{\mathrm{x}}$ Contact. Appl. Phys. Lett. 2014, 104, 113902.

[3] Wu, W.; Bao, J.; Jia, X.; Liu, Z.; Cai, L.; Liu, B.; Song, J.; Shen, H. Dopant-Free Back Contact Silicon Heterojunction Solar Cells Employing Transition Metal Oxide Emitters. Phys. Status Solidi RRL 2016, 10, 662-667.

[4] Gerling, L. G.; Mahato, S.; Morales-Vilches, A.; Masmitja, G.; Ortega, P.; Voz, C.; Alcubilla, R.; Puigdollers, J. Transition Metal Oxides as Hole-Selective Contacts in Silicon Heterojunctions Solar Cells. Sol. Energy Mater. Sol. Cells 2016, 145, 109-115.

[5] Bullock, J.; Hettick, M.; Geissbühler, J.; Ong, A. J.; Allen, T.; Sutter-Fella, C. M.; Chen, T.; Ota, H.; Schaler, E. W.; De Wolf, S.; et al. Efficient Silicon Solar Cells with Dopant-Free Asymmetric Heterocontacts. Nat. Energy 2016, 1, 1-7.

[6] Wan, Y.; Samundsett, C.; Bullock, J.; Allen, T.; Hettick, M.; Yan, D.; Zheng, P.; Zhang, X.; Cui, J.; McKeon, J.; et al. Magnesium Fluoride Electron-Selective Contacts for Crystalline Silicon Solar Cells. ACS Appl. Mater. Interfaces 2016, 8, 14671-14677. 
[7] Yang, X.; Liu, W.; De Bastiani, M.; Allen, T.; Kang, J.; Xu, H.; Aydin, E.; Xu, L.; Bi, Q.; Dang, H.; et al. Dual-Function Electron-Conductive, Hole-Blocking Titanium Nitride Contacts for Efficient Silicon Solar Cells. Joule 2019, 3, 1314-1327.

[8] Yang, Z.; Gao, P.; He, J.; Chen, W.; Yin, W.-Y.; Zeng, Y.; Guo, W.; Ye, J.; Cui, Y. Tuning of the Contact Properties for High-Efficiency Si/PEDOT:PSS Heterojunction Solar Cells. ACS Energy Lett. 2017, 2, 556-562.

[9] Liu, J.; Ji, Y.; Liu, Y.; Xia, Z.; Han, Y.; Li, Y.; Sun, B. Doping-Free Asymmetrical Silicon Heterocontact Achieved by Integrating Conjugated Molecules for High Efficient Solar Cell. Adv. Energy Mater. 2017, 7, 1700311.

[10] Holman, Z. C.; Descoeudres, A.; Barraud, L.; Fernandez, F. Z.; Seif, J. P.; De Wolf, S.;

Ballif, C. Current Losses at the Front of Silicon Heterojunction Solar Cells. IEEE J. Photovolt. 2012, 2, 7-15.

[11] Cuevas, A.; Basore, P. A.; Giroult-Matlakowski, G.; Dubois, C. Surface Recombination Velocity of Highly Doped N-Type Silicon. J. Appl. Phys. 1996, 80, 3370-3375.

[12] Yan, D.; Cuevas, A. Empirical Determination of the Energy Band Gap Narrowing in $P^{+}$ Silicon Heavily Doped with Boron. J. Appl. Phys. 2014, 116, 194505.

[13] Zhang, T.; Iqbal, S.; Zhang, X.-Y.; Wu, W.; Su, D.; Zhou, H.-L. Recent Advances in Highly Efficient Organic-Silicon Hybrid Solar Cells. Sol. Energy Mater. Sol. Cells 2020, 204, 110245.

[14] Zielke, D.; Pazidis, A.; Werner, F.; Schmidt, J. Organic-Silicon Heterojunction Solar Cells on N-Type Silicon Wafers: The BackPEDOT Concept. Sol. Energy Mater. Sol. Cells 2014, $131,110-116$.

[15] Ikeda, N.; Koganezawa, T.; Kajiya, D.; Saitow, K.-i. Performance of Si/PEDOT:PSS Hybrid Solar Cell Controlled by PEDOT:PSS Film Nanostructure. J. Phys. Chem. C 2016, 120, 19043-19048. 
[16] Jäckle, S.; Liebhaber, M.; Gersmann, C.; Mews, M.; Jäger, K.; Christiansen, S.; Lips, K. Potential of PEDOT:PSS as a Hole Selective Front Contact for Silicon Heterojunction Solar Cells. Sci. Rep. 2017, 7, 2170.

[17] Zielke, D.; Niehaves, C.; Lövenich, W.; Elschner, A.; Hörteis, M.; Schmidt, J. OrganicSilicon Solar Cells Exceeding 20\% Efficiency. Energy Procedia 2015, 77, 331-339.

[18] Tersoff, J. Schottky Barrier Heights and the Continuum of Gap States. Phys. Rev. Lett. $1984,52,465$.

[19] Tung, R. T. The Physics and Chemistry of the Schottky Barrier Height. Appl. Phys. Rev. 2014, $1,011304$.

[20] Sajjad, M.; Yang, X.; Altermatt, P.; Singh, N.; Schwingenschlögl, U.; De Wolf, S. MetalInduced Gap States in Passivating Metal/Silicon Contacts. Appl. Phys. Lett. 2019, 114, 071601. [21] He, J.; Wan, Y.; Gao, P.; Tang, J.; Ye, J. Over 16.7\% Efficiency Organic-Silicon Heterojunction Solar Cells with Solution-Processed Dopant-Free Contacts for Both Polarities. Adv. Funct. Mater. 2018, 28, 1802192.

[22] Zhang, Y.; Zu, F.; Lee, S. T.; Liao, L.; Zhao, N.; Sun, B. Heterojunction with Organic Thin Layers on Silicon for Record Efficiency Hybrid Solar Cells. Adv. Energy Mater. 2014, 4, 1300923.

[23] Wang, D.; Sheng, J.; Wu, S.; Zhu, J.; Chen, S.; Gao, P.; Ye, J. Tuning Back Contact Property via Artificial Interface Dipoles in Si/Organic Hybrid Solar Cells. Appl. Phys. Lett. 2016, $109,043901$.

[24] Reichel, C.; Würfel, U.; Winkler, K.; Schleiermacher, H.-F.; Kohlstädt, M.; Unmüssig, M.; Messmer, C. A.; Hermle, M.; Glunz, S. W. Electron-Selective Contacts via Ultra-Thin Organic Interface Dipoles for Silicon Organic Heterojunction Solar Cells. J. Appl. Phys. 2018, $123,024505$. 
[25] Zhang, Y.; Cui, W.; Zhu, Y.; Zu, F.; Liao, L.; Lee, S.-T.; Sun, B. High Efficiency Hybrid PEDOT:PSS/Nanostructured Silicon Schottky Junction Solar Cells by Doping-Free Rear Contact. Energy Environ. Sci. 2015, 8, 297-302.

[26] Mahmood, K.; Swain, B. S.; Amassian, A. 16.1\% Efficient Hysteresis-Free Mesostructured Perovskite Solar Cells Based on Synergistically Improved ZnO Nanorod Arrays. Adv. Energy Mater. 2015, 5, 1500568.

[27] Höfle, S.; Schienle, A.; Bruns, M.; Lemmer, U.; Colsmann, A. Enhanced Electron Injection into Inverted Polymer Light-Emitting Diodes by Combined Solution-Processed Zinc Oxide/Polyethylenimine Interlayers. Adv. Mater. 2014, 26, 2750-2754.

[28] Zhou, Y.; Fuentes-Hernandez, C.; Shim, J.; Meyer, J.; Giordano, A. J.; Li, H.; Winget, P.; Papadopoulos, T.; Cheun, H.; Kim, J. A Universal Method to Produce Low-Work Function Electrodes for Organic Electronics. Science 2012, 336, 327-332.

[29] Shim, M.; Javey, A.; Kam, N. W. S.; Dai, H. Polymer Functionalization for Air-Stable NType Carbon Nanotube Field-Effect Transistors. J. Am. Chem. Soc. 2001, 123, 11512-11513.

[30] Du, Y.; Liu, H.; Neal, A. T.; Si, M.; Ye, P. D. Molecular Doping of Multilayer $\mathrm{MoS}_{2}$ Field-Effect Transistors: Reduction in Sheet and Contact Resistances. IEEE Electron Device Lett. 2013, 34, 1328-1330.

[31] Huang, W.; Zeng, L.; Yu, X.; Guo, P.; Wang, B.; Ma, Q.; Chang, R. P.; Yu, J.; Bedzyk, M. J.; Marks, T. J.; et al. Metal Oxide Transistors via Polyethylenimine Doping of the Channel Layer: Interplay of Doping, Microstructure, and Charge Transport. Adv. Funct. Mater. 2016, 26, 6179-6187.

[32] Descoeudres, A.; Holman, Z. C.; Barraud, L.; Morel, S.; De Wolf, S.; Ballif, C. > 21\% Efficient Silicon Heterojunction Solar Cells on $N$-and $P$-Type Wafers Compared. IEEE J. Photovolt. 2012, 3, 83-89.

[33] Beamson, G.; Briggs, D. High Resolution XPS of Organic Polymers: The Scienta ESCA300 Database Wiley, Chichester, NY 1992. 
[34] Kim, Y. H.; Han, T. H.; Cho, H.; Min, S. Y.; Lee, C. L.; Lee, T. W. Polyethylene Imine as an Ideal Interlayer for Highly Efficient Inverted Polymer Light-Emitting Diodes. Adv. Funct. Mater. 2014, 24, 3808-3814.

[35] Allen, T. G.; Bullock, J.; Jeangros, Q.; Samundsett, C.; Wan, Y.; Cui, J.; Hessler-Wyser, A.; De Wolf, S.; Javey, A.; Cuevas, A. A Low Resistance Calcium/Reduced Titania Passivated Contact for High Efficiency Crystalline Silicon Solar Cells. Adv. Energy Mater. 2017, 7, 1602606.

[36] Ji, W.; Zhao, Y.; Fahad, H. M.; Bullock, J.; Allen, T.; Lien, D.-H.; De Wolf, S.; Javey, A. Dip Coating Passivation of Crystalline Silicon by Lewis Acids. ACS Nano 2019, 13, 3723-3729. [37] Karty, J. M. Organic Chemistry: Principles and Mechanisms Norton, New York, NY 2018. 